\title{
A Diagonally-Oriented DCT-Like 2D Block Transform
}

\author{
Ivan W. Selesnick ${ }^{a}$ and Onur G. Guleryuz ${ }^{b}$ \\ ${ }^{a}$ Polytechnic Institute of New York Univ., Brooklyn, NY 11201, USA \\ ${ }^{b}$ DoCoMo Communication Laboratories USA Inc, Palo Alto, CA 94304, USA
}

\begin{abstract}
Due to the prevalence of edges in image content, various directional transforms have been proposed for the efficient representation of images. Such transforms are useful for coding, denoising, and image restoration using sparse signal representation techniques. This paper describes a new non-separable 2D DCT-like orthonormal block transform that is optimized for a specified orientation angle. The approach taken in this paper is to extend to two-dimensions one approach (of several) for constructing the standard 1D DCT. The proposed transform is obtained as the eigenvectors of particular matrices, as is the standard 1D DCT.
\end{abstract}

Keywords: Discrete cosine transform, DCT, directional DCT, block transform

\section{INTRODUCTION}

Various directional linear transforms have been proposed for the efficient representation of images. Some directional transforms (steerable pyramids, curvelets, complex wavelets, shearlets, etc) consist of orientation-selective subbands and are effective for the efficient representation of almost arbitrary oriented image content. On the other hand, other directional transforms are optimized to a specific direction; for example several types of directional discrete cosine transforms (DCTs) have been proposed; see Refs. 2,3,7,8. As another example, a dictionary learning method for designing directional transforms from image patches has been proposed. ${ }^{4}$ For image processing and coding using a set of orientation-specific transforms, the transform used for a specific image block is selected depending on the orientation of that block.

This paper describes an approach for the design of oriented DCT-like block transforms that attempts to maintain certain properties of the one-dimensional DCT. In particular, Strang has noted that the basis functions of the $\mathrm{DCT}^{1}$ can be obtained exactly as the eigenvectors of a particular matrix. ${ }^{5,6}$ Viewed in another way, the DCT can be associated with the sequential constrained minimization of a specific cost function. To elaborate, consider the design of an orthonormal transform to be used to represent smooth signals; the transform should contain smooth basis functions. We can proceed with the design of the orthonormal basis by first finding the unit-norm vector that is maximally smooth (a constant function). This is the 'first' basis function of the basis. We then find the second basis function by finding the smoothest unit-norm vector among all vectors orthogonal to the first basis function. Next, we find the third basis function by finding the smoothest unit-norm vector among all vectors orthogonal to the first two, etc. When we use, as the measure of smoothness, the energy of the first-order difference, the result of this sequence of constrained optimization problems yields exactly the DCT.

We will use the same procedure to derive new directional DCT-like 2D orthonormal block transforms.

\section{DCT AS EIGENVECTORS}

Consider the design of a unit-norm eight-point sequence $x(n), n=1, \ldots, 8$, chosen so as to minimize the energy of the first-order difference of $\mathbf{x}=[x(1), \ldots, x(8)]^{t}$. We can write this as

$$
\min _{\mathbf{x}} \sum_{n=2}^{8}[x(n)-x(n-1)]^{2} \quad \text { subject to } \quad \mathbf{x}^{t} \mathbf{x}=1
$$

or as

$$
\min _{\mathbf{x}} J(\mathbf{x}) \quad \text { subject to } \quad \mathbf{x}^{t} \mathbf{x}=1
$$

Further author information: selesi@poly.edu.edu 
where the cost function $J(\mathbf{x})$ is given by

$$
J(\mathbf{x}):=\sum_{n=2}^{8}(x(n)-x(n-1))^{2}=\|\mathbf{A} \mathbf{x}\|_{2}^{2}=\mathbf{x}^{t} \mathbf{A}^{t} \mathbf{A} \mathbf{x}=\mathbf{x}^{t} \mathbf{R} \mathbf{x}
$$

where

$$
\mathbf{A}:=\left[\begin{array}{rrrrrrrr}
1 & -1 & 0 & 0 & 0 & 0 & 0 & 0 \\
0 & 1 & -1 & 0 & 0 & 0 & 0 & 0 \\
0 & 0 & 1 & -1 & 0 & 0 & 0 & 0 \\
0 & 0 & 0 & 1 & -1 & 0 & 0 & 0 \\
0 & 0 & 0 & 0 & 1 & -1 & 0 & 0 \\
0 & 0 & 0 & 0 & 0 & 1 & -1 & 0 \\
0 & 0 & 0 & 0 & 0 & 0 & 1 & -1
\end{array}\right]
$$

and

$$
\mathbf{R}:=\mathbf{A}^{t} \mathbf{A}\left[\begin{array}{rrrrrrrr}
1 & -1 & 0 & 0 & 0 & 0 & 0 & 0 \\
-1 & 2 & -1 & 0 & 0 & 0 & 0 & 0 \\
0 & -1 & 2 & -1 & 0 & 0 & 0 & 0 \\
0 & 0 & -1 & 2 & -1 & 0 & 0 & 0 \\
0 & 0 & 0 & -1 & 2 & -1 & 0 & 0 \\
0 & 0 & 0 & 0 & -1 & 2 & -1 & 0 \\
0 & 0 & 0 & 0 & 0 & -1 & 2 & -1 \\
0 & 0 & 0 & 0 & 0 & 0 & -1 & 1
\end{array}\right] \text {. }
$$

Of course, the unit-norm 8-point sequence $\mathbf{x}$ minimizing $J(\mathbf{x})$ is simply the 'constant' function, $x(n)=1 / \sqrt{8}$ for $1 \leq n \leq 8$ which gives $J(\mathbf{x})=0$. Let us call this sequence $\mathbf{x}_{1}=[1 / \sqrt{8}, \ldots, 1 / \sqrt{8}]^{t}$.

It turns out, that in the general case where $\mathbf{A}$ is arbitrary, the vector $\mathbf{x}$ minimizing $J(\mathbf{x})$ is given by the eigenvector of $\mathbf{A}^{t} \mathbf{A}$ having minimum eigenvalue. The derivation, using Lagrange multipliers, is given in Appendix A, with $\mathbf{K}=\mathbf{I}$ in that appendix.

Now, let us find another unit-norm 8-point sequence, we will call it $\mathbf{x}_{2}$, minimizing $J(\mathbf{x})$ but which is orthogonal to the vector just found, $\mathbf{x}_{1}$. We can write

$$
\min _{\mathbf{x}} \mathbf{x}^{t} \mathbf{A}^{t} \mathbf{A} \mathbf{x} \quad \text { subject to } \mathbf{x}^{t} \mathbf{x}=1 \text { and } \mathbf{x}_{1}^{t} \mathbf{x}=0 .
$$

It turns out that the solution $\mathbf{x}$ is the eigenvector of $\mathbf{A}^{t} \mathbf{A}$ corresponding to the next smallest eigenvalue. Proceeding similarly, we look for a vector $\mathbf{x}_{3}$ minimizing $J(\mathbf{x})$ but which is orthogonal to the previous two vectors; this optimization problem is stated as

$$
\min _{\mathbf{x}} \mathbf{x}^{t} \mathbf{A}^{t} \mathbf{A} \mathbf{x} \quad \text { subject to } \mathbf{x}^{t} \mathbf{x}=1 \text { and }\left[\mathbf{x}_{1}, \mathbf{x}_{2}\right]^{t} \mathbf{x}=\mathbf{0}
$$

where $\mathbf{x}_{1}$ and $\mathbf{x}_{2}$ are eigenvectors of $\mathbf{A}^{t} \mathbf{A}$. The solution is the next eigenvector of $\mathbf{A}^{t} \mathbf{A}$.

In general, if $\mathbf{x}_{1}, \ldots, \mathbf{x}_{m}$ are a given set of eigenvectors of $\mathbf{A}^{t} \mathbf{A}$, the problem of finding the unit-norm vector $\mathbf{x}$ minimizing $J(\mathbf{x})$ but which is orthogonal to the given eigenvectors, can be written as:

$$
\min _{\mathbf{x}} \mathbf{x}^{t} \mathbf{A}^{t} \mathbf{A} \mathbf{x} \quad \text { subject to } \mathbf{x}^{t} \mathbf{x}=1 \text { and }\left[\mathbf{x}_{1}, \mathbf{x}_{2}, \cdots, \mathbf{x}_{m}\right]^{t} \mathbf{x}=\mathbf{0} \text {. }
$$

The solution $\mathbf{x}$ is the eigenvector of $\mathbf{A}^{t} \mathbf{A}$ corresponding to the next smallest eigenvalue of $\mathbf{A}^{t} \mathbf{A}$. This is shown in Appendix B, with $\mathbf{K}=\mathbf{I}$ in that appendix,

According to the forgoing discussion, we may find the solution to this sequence of constrained optimization problems by simply computing the eigenvector decomposition of the matrix $\mathbf{R}:=\mathbf{A}^{t} \mathbf{A}$,

$$
\mathbf{R}=\mathbf{V} \mathbf{D} \mathbf{V}^{t}
$$

where $\mathbf{D}$ is diagonal matrix and $\mathbf{V}$ is an orthonormal matrix, $\mathbf{V}^{-1}=\mathbf{V}^{T}$. It turns out ${ }^{5}$ that for the matrix $\mathbf{R}$ in (2), the eigenvectors (the columns of $\mathbf{V}$ ) are exactly the basis functions of the DCT, illustrated in Fig. 1. 

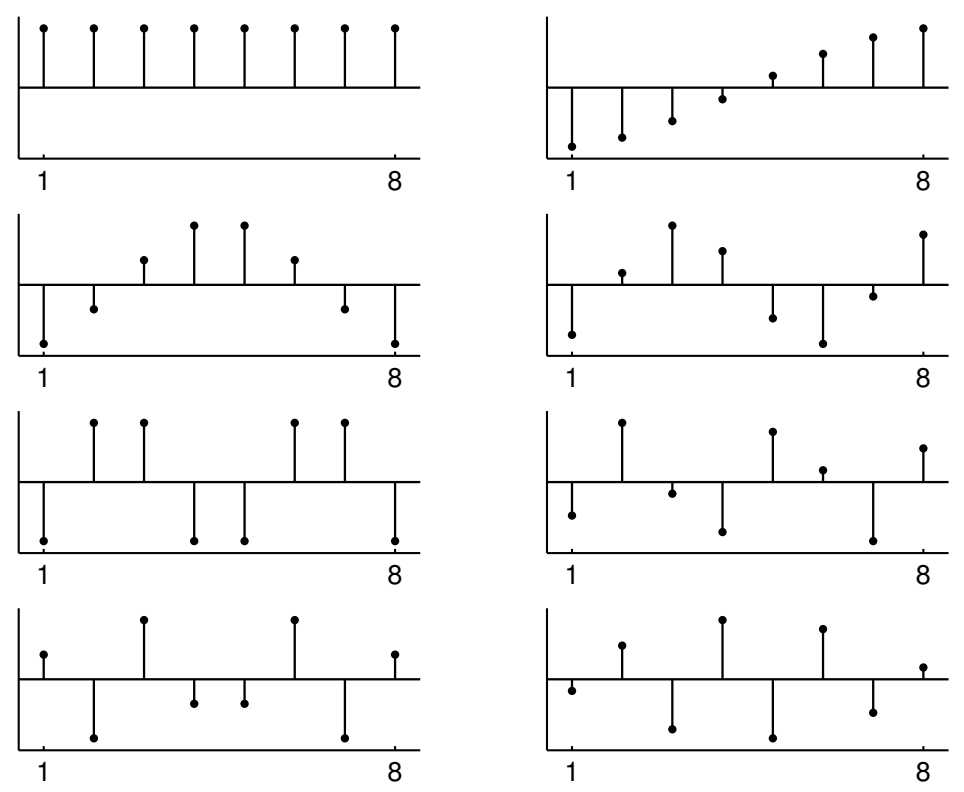

Figure 1. Basis functions of the eight-point DCT.

\section{DIAGONALLY-ORIENTED 2D BASIS FUNCTIONS}

Our goal is the design of a diagonally-oriented 2D orthonormal block transform. It appears reasonable that several of the $2 \mathrm{D}$ basis functions of such a transform should be constant along all their diagonals. That is to say, for an $8 \times 8$ block transform, some of the basis functions should have the structure:

$$
\mathbf{b}=\left[\begin{array}{cccccccc}
x_{1} & x_{2} & x_{3} & x_{4} & x_{5} & x_{6} & x_{7} & x_{8} \\
x_{2} & x_{3} & x_{4} & x_{5} & x_{6} & x_{7} & x_{8} & x_{9} \\
x_{3} & x_{4} & x_{5} & x_{6} & x_{7} & x_{8} & x_{9} & x_{10} \\
x_{4} & x_{5} & x_{6} & x_{7} & x_{8} & x_{9} & x_{10} & x_{11} \\
x_{5} & x_{6} & x_{7} & x_{8} & x_{9} & x_{10} & x_{11} & x_{12} \\
x_{6} & x_{7} & x_{8} & x_{9} & x_{10} & x_{11} & x_{12} & x_{13} \\
x_{7} & x_{8} & x_{9} & x_{10} & x_{11} & x_{12} & x_{13} & x_{14} \\
x_{8} & x_{9} & x_{10} & x_{11} & x_{12} & x_{13} & x_{14} & x_{15}
\end{array}\right] .
$$

In order to construct an orthonormal set of $2 \mathrm{D}$ basis functions of this form, we can utilize the sequential constrained optimization process that gives rise to the 1D DCT. However, a few modifications are required (primarily, the need for the matrix $\mathbf{K}$ in the following).

The $2 \mathrm{D}$ basis function $\mathbf{b}$ can be represented by a vector $\mathbf{x}$,

$$
\mathbf{x}=[x(1), \ldots, x(15)]^{t}
$$

Let us use the cost function,

$$
J(\mathbf{x})=\sum_{n=2}^{15}[x(n)-x(n-1)]^{2}=\|\mathbf{A} \mathbf{x}\|_{2}^{2}=\mathbf{x}^{t} \mathbf{A}^{t} \mathbf{A} \mathbf{x}=\mathbf{x}^{t} \mathbf{R} \mathbf{x}
$$

where $\mathbf{A}$ and $\mathbf{R}$ are as in (1) and (2), only larger in size. This cost function penalizes changes in values between adjacent diagonals.

Note that the norm of $\mathbf{b}$ can be written as

$$
\|\mathbf{b}\|_{2}^{2}=\mathbf{x}^{t} \mathbf{K} \mathbf{x}
$$


where $\mathbf{K}$ is the diagonal matrix

$$
\mathbf{K}=\operatorname{diag}([1,2,3,4,5,6,7,8,7,6,5,4,3,2,1]) .
$$

Therefore, finding the unit-norm $2 \mathrm{D}$ basis function $\mathbf{b}$ minimizing the cost function $J$, requires solving the constrained optimization problem

$$
\min _{\mathbf{x}} \mathbf{x}^{t} \mathbf{A}^{t} \mathbf{A} \mathbf{x} \quad \text { subject to } \quad \mathbf{x}^{t} \mathbf{K} \mathbf{x}=1
$$

As shown in Appendix A, the minimizing vector $\mathbf{x}$ is given by the generalized eigenvector of $\left(\mathbf{A}^{t} \mathbf{A}, \mathbf{K}\right)$ corresponding to the minimum eigenvalue. For our $\mathbf{A}$, the minimizing vector $\mathbf{x}$ is, as expected, the constant function, $x(n)=1 / 8$, for $1 \leq n \leq 15$. So the first $2 \mathrm{D}$ basis function, denoted as $\mathbf{b}_{1}$, is the constant function.

Next, we would like to find a second unit-norm $2 \mathrm{D}$ basis function minimizing $J(\mathbf{x})$ but which is orthogonal to the first basis function. Note that if $\mathbf{b}_{1}$ and $\mathbf{b}_{2}$ are $2 \mathrm{D}$ basis functions having the structure shown in (3), then they are orthogonal if

$$
\mathbf{x}_{1}^{t} \mathbf{K} \mathbf{x}_{2}=0 .
$$

Therefore, to find the second unit-norm $2 \mathrm{D}$ basis function $\mathbf{b}_{2}$, we need to solve,

$$
\min _{\mathbf{x}} \mathbf{x} \mathbf{A}^{t} \mathbf{A} \mathbf{x} \quad \text { subject to } \mathbf{x}^{t} \mathbf{K} \mathbf{x}=1 \text { and } \mathbf{x}_{1}^{t} \mathbf{K} \mathbf{x}=\mathbf{0} .
$$

As shown in Appendix B, the solution is the generalized eigenvector of $\left(\mathbf{A}^{t} \mathbf{A}, \mathbf{K}\right)$ corresponding to the second smallest eigenvalue.

Similar to what we saw in Section 2, if $\mathbf{x}_{1}, \ldots, \mathbf{x}_{m}$ are a given set of generalized eigenvectors of $\left(\mathbf{A}^{t} \mathbf{A}, \mathbf{K}\right)$, then the problem of finding the unit-norm vector $\mathbf{x}$ minimizing $J(\mathbf{x})$ but which is $\mathbf{K}$-orthogonal to the given eigenvectors, can be written as:

$$
\min _{\mathbf{x}} \mathbf{x} \mathbf{A}^{t} \mathbf{A} \mathbf{x} \quad \text { subject to } \quad \mathbf{x}^{t} \mathbf{K} \mathbf{x}=1 \quad \text { and } \quad\left[\mathbf{x}_{1}, \mathbf{x}_{2}, \cdots, \mathbf{x}_{m}\right]^{t} \mathbf{K x}=\mathbf{0} .
$$

The solution $\mathbf{x}$ is the generalized eigenvector of $\left(\mathbf{A}^{t} \mathbf{A}, \mathbf{K}\right)$ corresponding to the next smallest eigenvalue. This is shown in Appendix B.

According to the forgoing discussion, we may find the solution to this sequence of constrained optimization problems by simply computing the generalized eigenvector decomposition

$$
\mathbf{R V}=\mathbf{K V D}
$$

where $\mathbf{D}$ is diagonal matrix and $\mathbf{V}$ satisfies $\mathbf{V}^{t} \mathbf{K V}=\mathbf{I}$.

For $\mathbf{K}$ in (4) and $\mathbf{A}$ in (1), the resulting vectors are illustrated in Fig. 2. Notice that roughly the first half of these vectors resemble the basis functions of the DCT - the second basis function resembles half a period of a cosine function, the third basis function resembles a whole period of a cosine, etc. However, these are not exactly cosine functions. The last few functions are quite different from DCT basis functions - the last few functions are localized at both ends which is not desirable for efficient representation of image blocks. In the following section, we will use just the first eight of these fifteen functions.

The 15-point $\mathbf{K}$-orthonormal vectors illustrated in Fig. 2 yield the $8 \times 82 \mathrm{D}$ orthonormal basis functions illustrated in Fig. 3. To construct an orthonormal basis for $8 \times 8$ blocks, we need 64 2D basis functions. Therefore, we need to find additional 2D basis functions that are orthogonal to the ones shown in Fig. 3 (or to the subset thereof which we wish to employ).

\section{2D DCT AS EIGENVECTORS}

The 2D DCT is usually implemented and derived as a row-column separable transform. However, our goal here is to develop a (non-separable) DCT-like 2D transform. Therefore, it is informative to examine a derivation the 2D DCT that does not utilize row-column separability. It turns out, the sequential constrained optimization procedure that yields the 1D DCT (as described in Section 2) can be applied directly in 2D. 

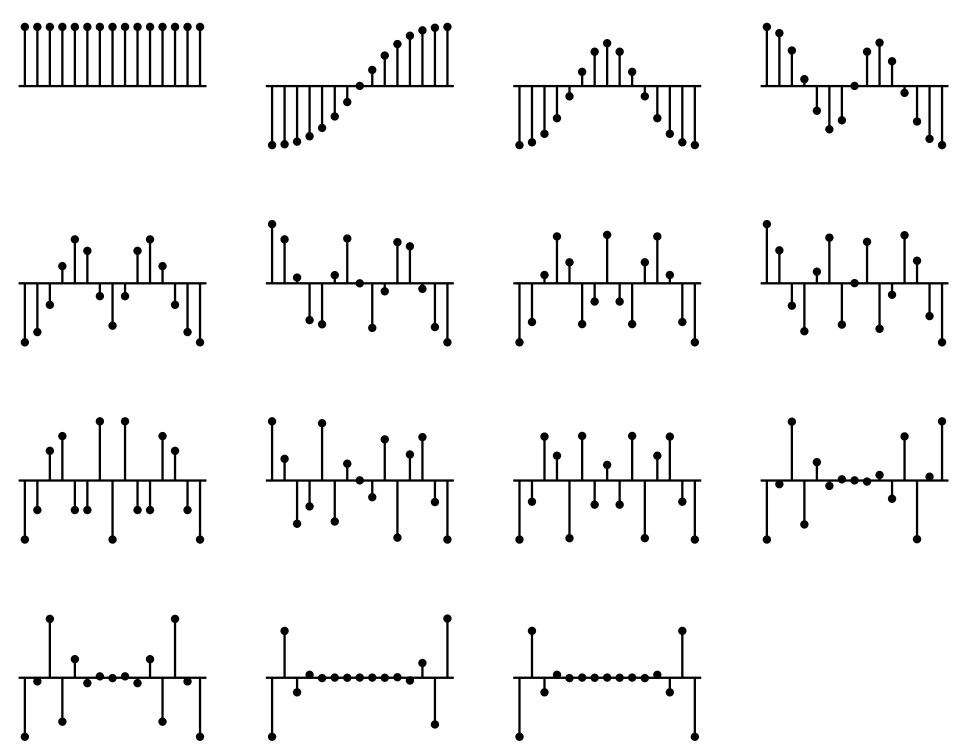

Figure 2. 15-point DCT-like transform. The basis functions are orthonormal with respect to $\mathbf{K}$ in (4). They are obtained as generalized eigenvectors, see (5).

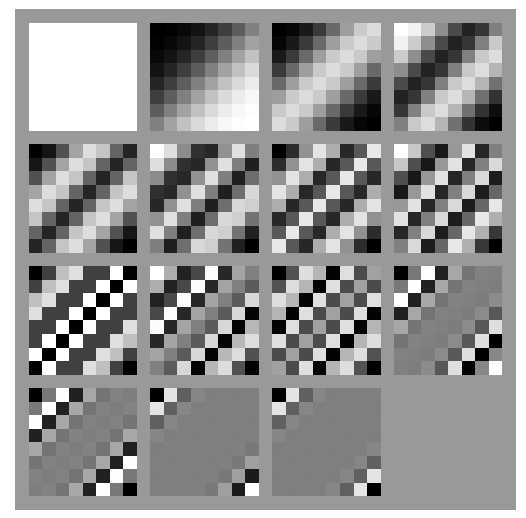

Figure 3. Orthonormal 2D basis functions. Each basis function is constant along all of its diagonals. 
Consider the vector $\mathbf{x}$ to be a 'vectorization' of the $8 \times 82 \mathrm{D}$ array, $x\left(n_{1}, n_{2}\right)$ for $1 \leq n_{1}, n_{2}, \leq 8$.

Define the cost function

$$
J(\mathbf{x})=c_{h}^{2} \sum_{n_{1}=2}^{8} \sum_{n_{2}=1}^{8}\left[x\left(n_{1}, n_{2}\right)-x\left(n_{1}-1, n_{2}\right)\right]^{2}+c_{v}^{2} \sum_{n_{1}=1}^{8} \sum_{n_{2}=2}^{8}\left[x\left(n_{1}, n_{2}\right)-x\left(n_{1}, n_{2}-1\right)\right]^{2} .
$$

This cost function is the sum of the energy of the horizontal first-order difference and the energy of the vertical first-order difference. The cost function can be written as $J(\mathbf{x})=\left\|c_{h} \mathbf{A}_{h} \mathbf{x}\right\|_{2}^{2}+\left\|c_{v} \mathbf{A}_{v} \mathbf{x}\right\|_{2}^{2}$ where $\mathbf{A}_{h}$ represents the horizontal difference operator, and $\mathbf{A}_{v}$ represents the vertical difference operator. Therefore, we can write the cost function as

$$
J(\mathbf{x})=\mathbf{x}^{t} \mathbf{A}^{t} \mathbf{A} \mathbf{x} \quad \text { where } \quad \mathbf{A}=\left[\begin{array}{c}
c_{h} \mathbf{A}_{h} \\
c_{v} \mathbf{A}_{v}
\end{array}\right] .
$$

To find an orthonormal 2D basis, we can find the eigenvectors of $\mathbf{A}^{t} \mathbf{A}$.

With $c_{v}=1$ and $c_{h}=0.1$, we obtain the orthonormal 2D basis illustrated in Fig. 4a. The eigenvalues of $\mathbf{A}^{t} \mathbf{A}$ are illustrated in Fig. 4b. Note that here we have penalized vertical differences much more than horizontal differences $\left(c_{v}=10 c_{h}\right)$, so the first basis functions, corresponding to the smallest eigenvalues, are all verticallyoriented. (The top row in Fig. 4a are the basis functions corresponding to the eight lowest eigenvalues.) Also, notice that the eigenvalues are well-separated in value; the ninth basis function is the first one to have vertical variation, and the ninth eigenvalue is substantially higher than the eighth, etc.

With $c_{v}=1$ and $c_{h}=0.8$, we obtain the orthonormal 2D basis and eigenvalues illustrated in Figs. 4c and $4 \mathrm{~d}$. Note that here vertical and horizontal differences are penalized quite similarly so the ordering of the basis functions is quite different than that in Fig. 4a. The vertical and horizontal basis functions are interleaved more evenly, similar to the zig-zag ordering of 2D DCT coefficients in JPEG.

\section{COMPLETING THE DIAGONALLY-ORIENTED 2D BASIS FUNCTIONS}

In Section 3 we derived an orthonormal set of $2 \mathrm{D}$ functions, where each $2 \mathrm{D}$ function is constant along each of its diagonals. In this section, we wish to complete this set of basis functions to a full orthonormal basis for $8 \times 8$ blocks. Following the 2D DCT as a guide, we wish to utilize the cost function in (6) in Section 4.

In order to complete the orthonormal basis, we can find a sequence of $2 \mathrm{D}$ basis functions where each basis function minimizes a specified cost function but is also orthogonal to the previously found basis functions. Let $\mathbf{b}_{1}, \ldots, \mathbf{b}_{m}$ represent an orthonormal set of $2 \mathrm{D}$ basis functions already found. Then we can find a new $2 \mathrm{D}$ basis function by solving the constrained optimization problem,

$$
\min _{\mathbf{x}} \mathbf{x} \mathbf{A}^{t} \mathbf{A} \mathbf{x} \quad \text { subject to } \mathbf{x}^{t} \mathbf{x}=1 \text { and }\left[\mathbf{b}_{1}, \mathbf{b}_{2}, \cdots, \mathbf{b}_{m}\right]^{t} \mathbf{x}=\mathbf{0}
$$

where $\mathbf{A}$ is the matrix in (6) and where $\mathbf{b}_{i}$ and $\mathbf{x}$ represent vectorized forms of $2 \mathrm{D}$ basis functions. Similarly, a sequence of such optimization problems can be formulated so as to find a complete orthonormal basis.

The solution vectors to this sequence of optimizations problems can be found as $\mathbf{C u}_{i}$ where $\mathbf{u}_{i}$ are the generalized eigenvectors of $\left(\mathbf{C}^{t} \mathbf{A}^{t} \mathbf{A C}, \mathbf{C}^{t} \mathbf{C}\right)$ where $\mathbf{C}$ is a basis for the null space of $\mathbf{B}^{t}=\left[\mathbf{b}_{1}, \mathbf{b}_{2}, \cdots, \mathbf{b}_{m}\right]^{t}$, as described in Appendix C.

Starting with the first eight of the fifteen diagonally-oriented 2D basis functions illustrated in Fig. 3, the completion to an orthonormal 2D basis yields the sixty-four orthonormal basis functions illustrated in Fig. 5. This basis may be considered a diagonally-oriented 2D DCT-like transform.

\section{2D BASIS WITH OTHER ORIENTATION ANGLES}

The approach we have used above, can not produce transforms optimized for arbitrary orientation angles; however, in can be extended to some orientations. For example, if the slope of the orientation is taken to be one half (26.56 degrees), then the approach can be used. 


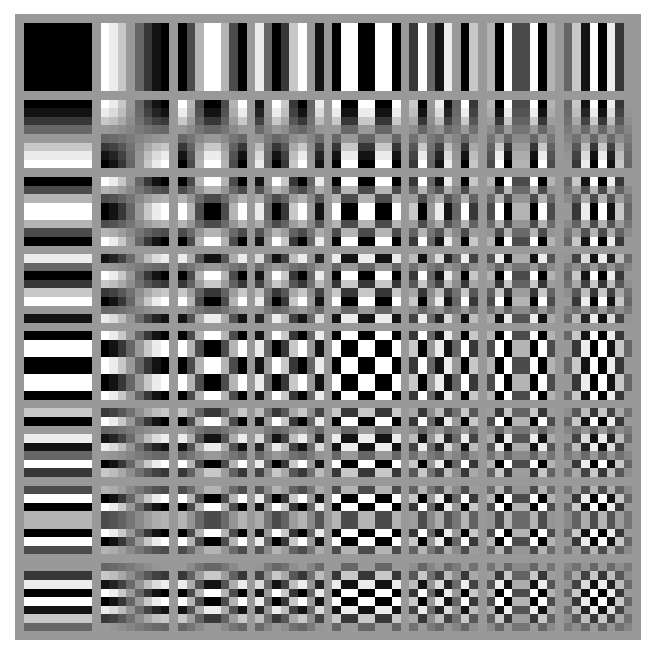

(a) Ortho-basis. $c_{v}=1, c_{h}=0.1$

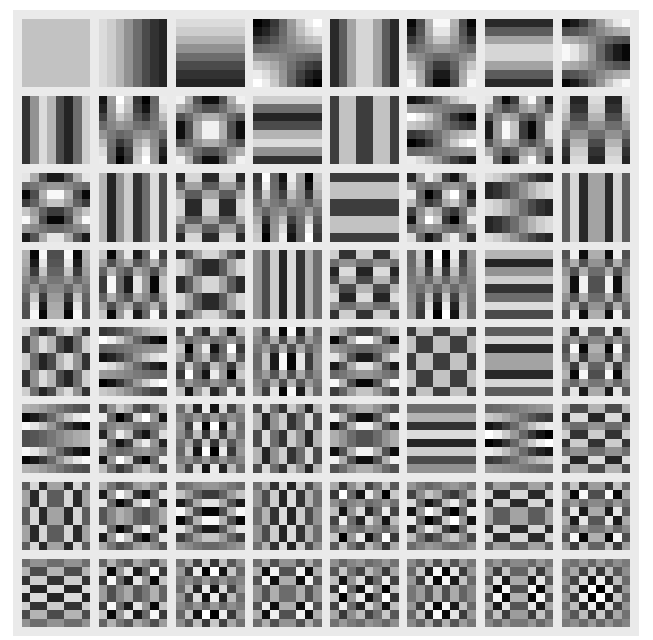

(c) Ortho-basis. $c_{v}=1, c_{h}=0.8$

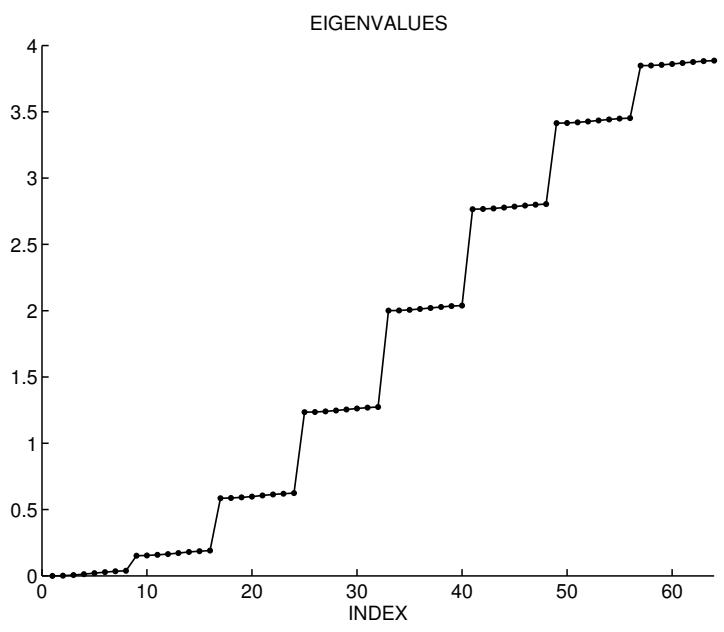

(b) Eigenvalues corresp. (a)

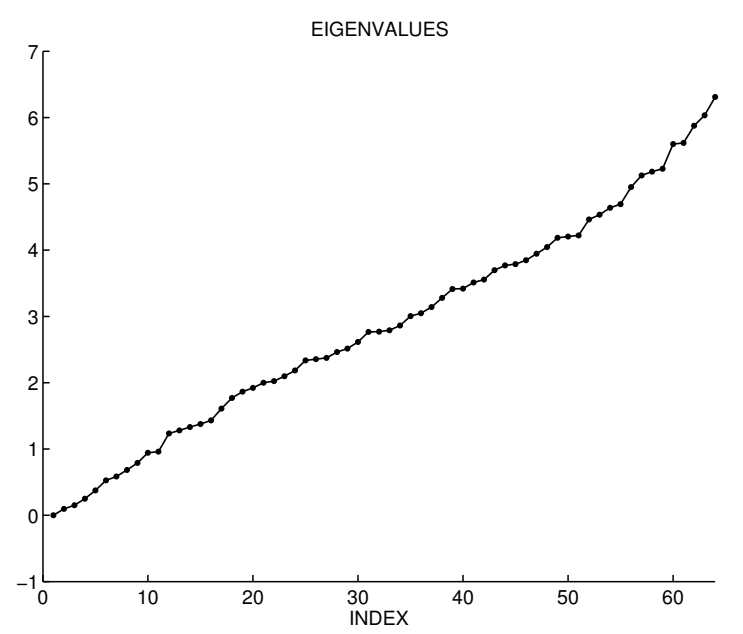

(d) Eigenvalues corresp. (c)

Figure 4. Orthonormal 2D basis functions obtained by minimizing $J(\mathbf{x})$ in (6). 


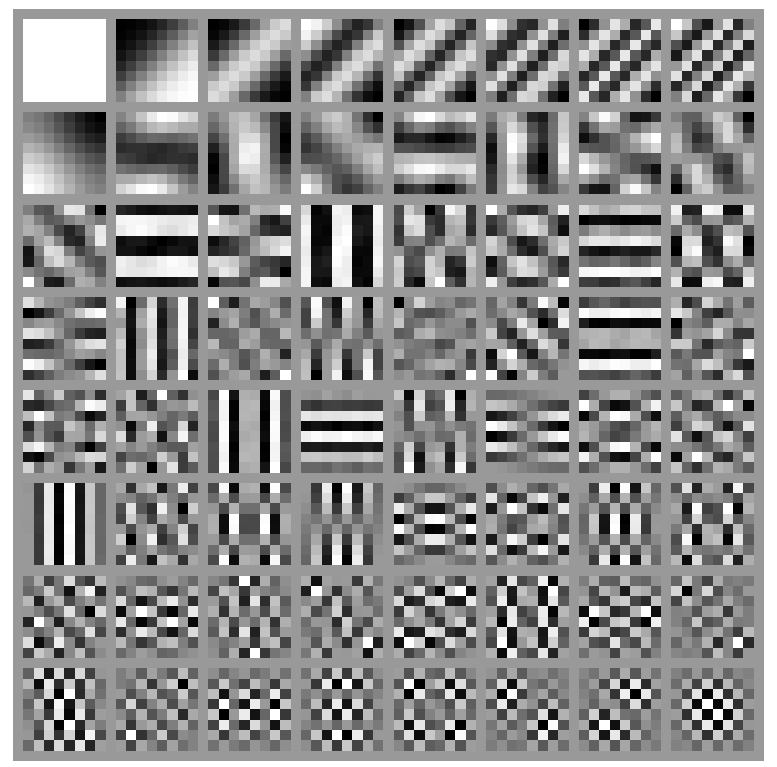

Figure 5. Orthonormal $8 \times 82 \mathrm{D}$ basis. The first eight basis functions are constant along their diagonals. The remaining basis functions complete the basis by minimizing the cost function in (6) subject to orthogonality constraints.

\subsection{Orientation Slope of $1 / 2$}

By asking that the 2D transform contain smooth basis functions with the structure,

$$
\mathbf{b}=\left[\begin{array}{cccccccc}
x_{1} & x_{2} & x_{3} & x_{4} & x_{5} & x_{6} & x_{7} & x_{8} \\
x_{3} & x_{4} & x_{5} & x_{6} & x_{7} & x_{8} & x_{9} & x_{10} \\
x_{5} & x_{6} & x_{7} & x_{8} & x_{9} & x_{10} & x_{11} & x_{12} \\
x_{7} & x_{8} & x_{9} & x_{10} & x_{11} & x_{12} & x_{13} & x_{14} \\
x_{9} & x_{10} & x_{11} & x_{12} & x_{13} & x_{14} & x_{15} & x_{16} \\
x_{11} & x_{12} & x_{13} & x_{14} & x_{15} & x_{16} & x_{17} & x_{18} \\
x_{13} & x_{14} & x_{15} & x_{16} & x_{17} & x_{18} & x_{19} & x_{20} \\
x_{15} & x_{16} & x_{17} & x_{18} & x_{19} & x_{20} & x_{21} & x_{22}
\end{array}\right]
$$

we obtain a $2 \mathrm{D}$ transform optimized for orientation slopes of $1 / 2$, that is 26.5 degrees. Each pixel is equal in value to the pixel two samples to the right and one sample above. It can be seen that there are 22 independent pixel values; therefore, the set of all such $8 \times 8$ blocks is a 22-dimensional subspace.

We can construct a basis for this this 22-dimensional subspace by sequential minimization with orthogonality conditions. As above, it is equivalent to computing a set of eigenvectors. In this case, $\mathbf{K}$ is given by

$$
\mathbf{K}=\operatorname{diag}([1,1,2,2,3,3,4,4,4,4,4,4,4,4,4,4,3,3,2,2,1,1]) .
$$

The resulting $1 \mathrm{D}$ functions, and the 2D functions to which they are mapped, are illustrated in Fig. 6. As above, the later vectors are localized at both endpoints which is not desirable for efficient image representation. We can also complete the set to a full orthonormal basis for $8 \times 8$ blocks, as illustrated in Fig. 7 . 

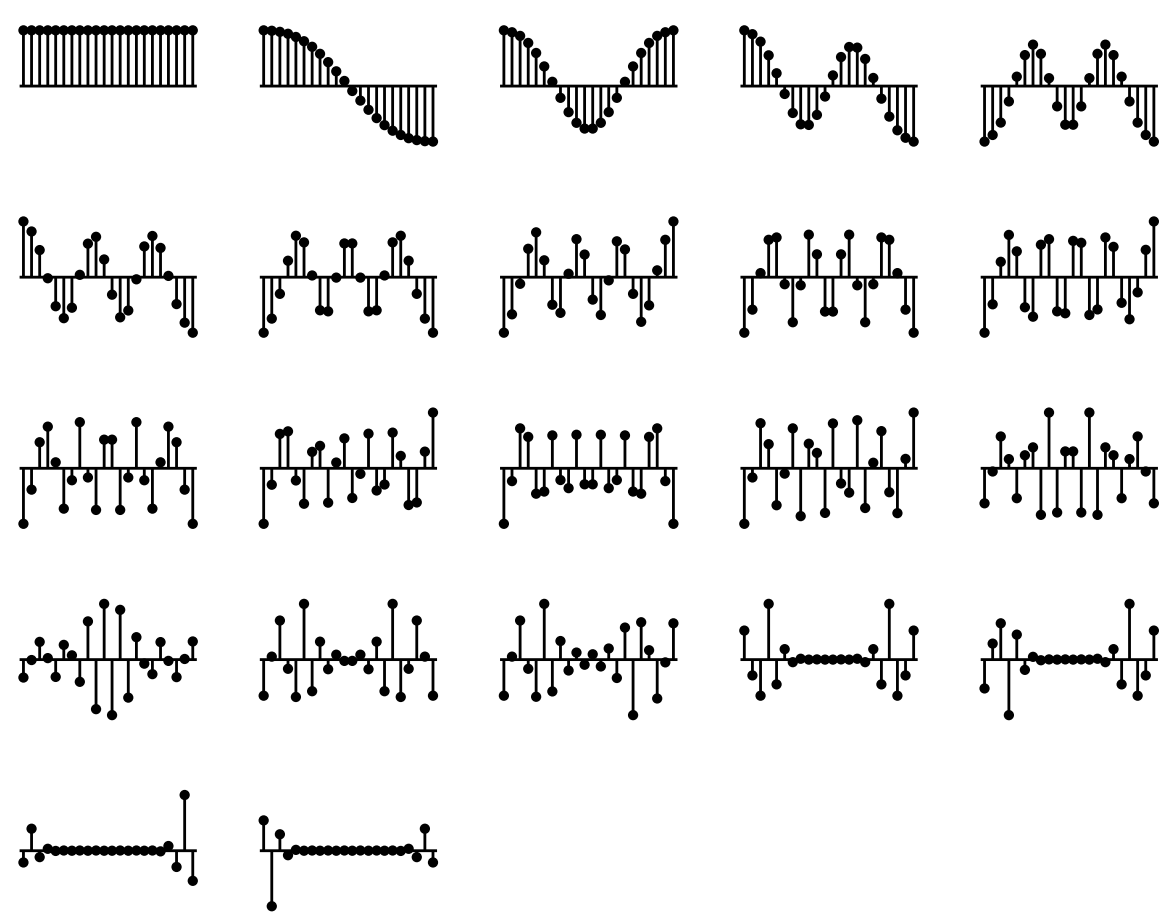

(a) 1D basis orthogonal with respect to $\mathbf{K}$ in (9).

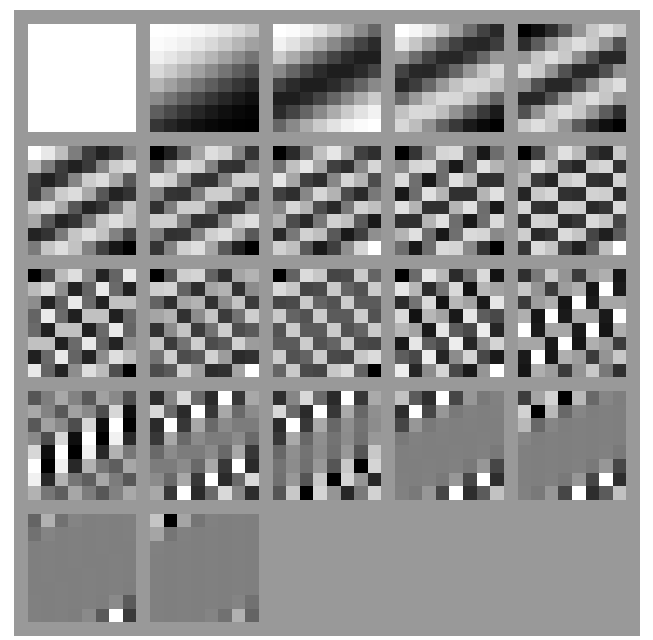

(b) $2 \mathrm{D}$ orthonormal basis for subpace of $8 \times 8$ blocks.

Figure 6. Constrained pixels, slope $=1 / 2$. 


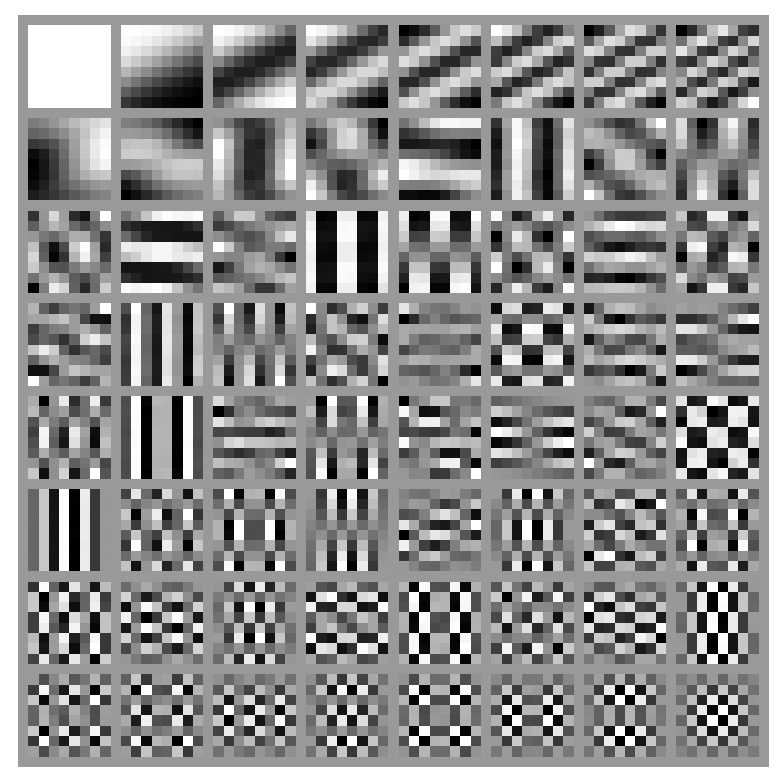

Figure 7. Orthonormal $8 \times 82 \mathrm{D}$ basis with orientation angle of 26.5 degrees (corresp. to an orientation slope of 0.5 ). The first eight basis functions have the structure given in (8). The remaining basis functions complete the basis by minimizing the cost function in (6) subject to orthogonality constraints. Compare to Fig. 5.

\subsection{Orientation Slope of $1 / 3$}

By asking that the 2D transform contain smooth basis functions with the structure,

$$
\mathbf{b}=\left[\begin{array}{cccccccc}
x_{1} & x_{2} & x_{3} & x_{4} & x_{5} & x_{6} & x_{7} & x_{8} \\
x_{4} & x_{5} & x_{6} & x_{7} & x_{8} & x_{9} & x_{10} & x_{11} \\
x_{7} & x_{8} & x_{9} & x_{10} & x_{11} & x_{12} & x_{13} & x_{14} \\
x_{10} & x_{11} & x_{12} & x_{13} & x_{14} & x_{15} & x_{16} & x_{17} \\
x_{13} & x_{14} & x_{15} & x_{16} & x_{17} & x_{18} & x_{19} & x_{20} \\
x_{16} & x_{17} & x_{18} & x_{19} & x_{20} & x_{21} & x_{22} & x_{23} \\
x_{19} & x_{20} & x_{21} & x_{22} & x_{23} & x_{24} & x_{25} & x_{26} \\
x_{22} & x_{23} & x_{24} & x_{25} & x_{26} & x_{27} & x_{28} & x_{29}
\end{array}\right]
$$

we obtain a $2 \mathrm{D}$ transform optimized for orientation slopes of $1 / 3$, that is 18.4 degrees. The matrix $\mathbf{K}$ is given by

$$
\mathbf{K}=\operatorname{diag}([1,1,1,2,2,2,3,3,2,3,3,2,3,3,2,3,3,2,3,3,2,3,3,2,2,2,1,1,1]) .
$$

The resulting orthonormal 2D basis, after completion to a full set of 64 functions, is illustrated in Fig. 8 .

\subsection{Orientation Slope of $2 / 3$}

By asking that the $2 \mathrm{D}$ transform contain smooth basis functions with the structure,

$$
\mathbf{b}=\left[\begin{array}{cccccccc}
x_{1} & x_{3} & x_{5} & x_{7} & x_{9} & x_{11} & x_{13} & x_{15} \\
x_{4} & x_{6} & x_{8} & x_{10} & x_{12} & x_{14} & x_{16} & x_{18} \\
x_{7} & x_{9} & x_{11} & x_{13} & x_{15} & x_{17} & x_{19} & x_{21} \\
x_{10} & x_{12} & x_{14} & x_{16} & x_{18} & x_{20} & x_{22} & x_{24} \\
x_{13} & x_{15} & x_{17} & x_{19} & x_{21} & x_{23} & x_{25} & x_{27} \\
x_{16} & x_{18} & x_{20} & x_{22} & x_{24} & x_{26} & x_{28} & x_{30} \\
x_{19} & x_{21} & x_{23} & x_{25} & x_{27} & x_{29} & x_{31} & x_{33} \\
x_{22} & x_{24} & x_{26} & x_{28} & x_{30} & x_{32} & x_{34} & x_{36}
\end{array}\right]
$$




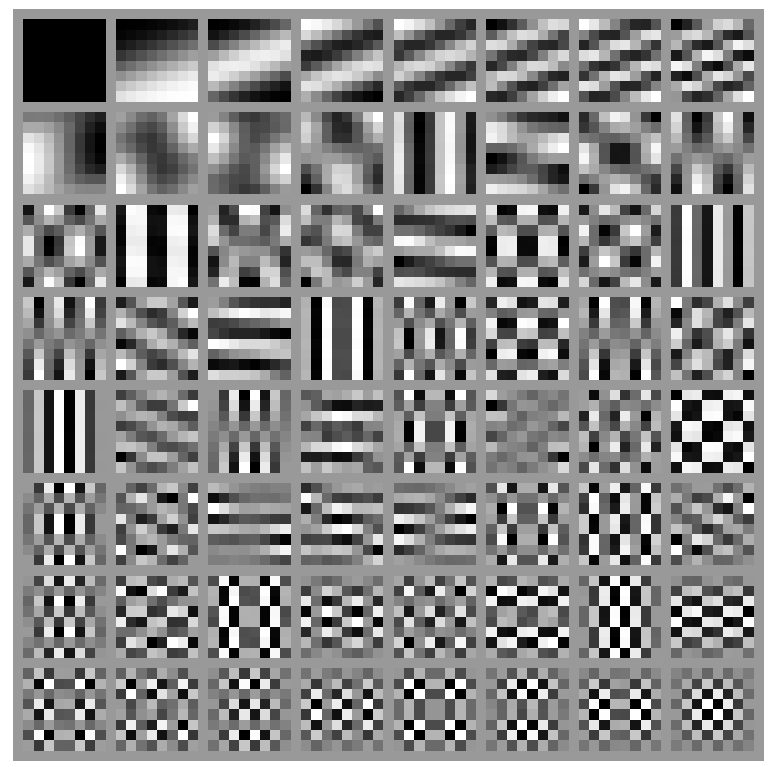

Figure 8 . Orthonormal $8 \times 82 \mathrm{D}$ basis with orientation angle of 18.4 degrees (corresp. to an orientation slope of $1 / 3$ ). The construction uses $\mathbf{K}$ in (10) and the same procedure as above.

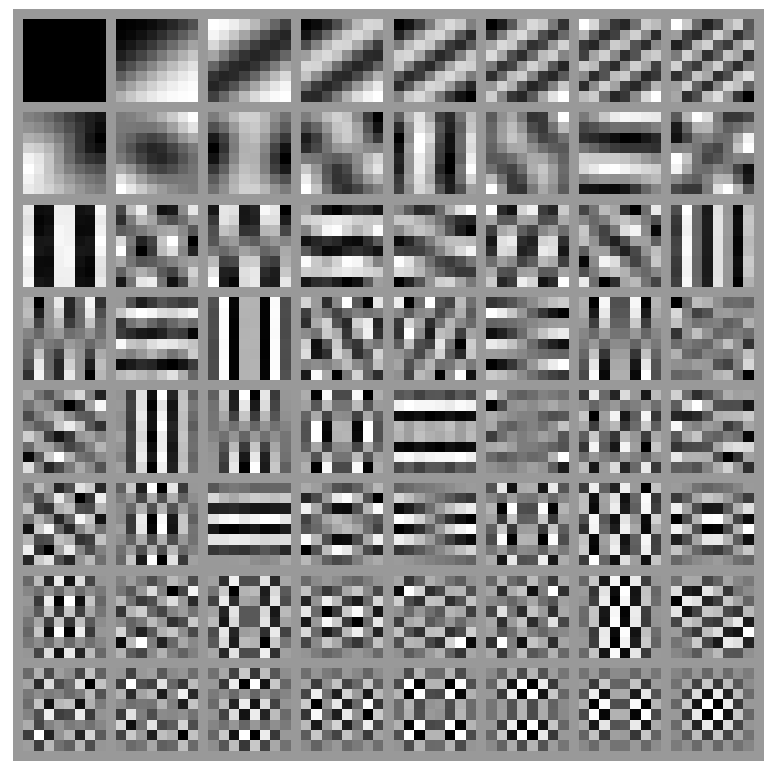

Figure 9. Orthonormal $8 \times 82 \mathrm{D}$ basis with orientation angle of 33.7 degrees (corresp. to an orientation slope of $2 / 3$ )

we obtain a $2 \mathrm{D}$ transform optimized for orientation slopes of $2 / 3$, that is 33.7 degrees. The $\mathbf{b}(i, j)$ entry of $\mathbf{b}$ is given by the formula

$$
\mathbf{b}(i, j)=x_{3 i+2 j-4}, \quad 1 \leq i, j, \leq 8
$$

Note that, unlike the previous examples, two elements of the matrix $\mathbf{K}$ are zero (second and second last location),

$$
\mathbf{K}=\operatorname{diag}([1,0,1,1,1,1,2,1,2,2,2,2,3,2,3,3,2,3,3,2,3,3,2,3,2,2,2,2,1,2,1,1,1,1,0,1])
$$

However, it is not an issue - the procedure still yields a basis with the desired properties. The resulting orthonormal 2D basis, after completion to a full set of 64 functions, is illustrated in Fig. 9. 


\section{CONCLUSION}

This paper presents a simple approach to produce directional orthonormal block transforms that resemble in a sense the classical 2D DCT. The primary basis functions are structurally directional, due to equality constraints among pixels in a specified orientation. The method can be used to generate a set of orthonormal transforms (one for each orientation) so that different blocks in an image can be processed using different transforms according to the dominant orientation of the block. The transform is optimal in a sense similar to the DCT — the design is based on the minimization of an object function maximizing smoothness, and the basis functions are obtained via generalized eigenvectors. The resulting transforms are truly non-separable (there are no row/col/grid issues arising from applying 1D transforms along specified orientations). The proposed method is easily extended (to other block shapes/size, higher dimensions, etc).

Note, however, unlike the classical DCT, the proposed transform has no explicit functional form and has no fast algorithm. In addition, the orientation angle not continuously variable and nor does it have a multiscale property, which can be desirable.

\section{APPENDIX A. MINIMIZATION WITH WEIGHTED INNER PRODUCT}

Consider the cost function is given by

$$
J(\mathbf{x})=\mathbf{x}^{t} \mathbf{A}^{t} \mathbf{A} \mathbf{x},
$$

and consider the constrained minimization problem:

$$
\min _{\mathbf{x}} J(\mathbf{x}) \quad \text { subject to } \quad \mathbf{x}^{t} \mathbf{K} \mathbf{x}=1
$$

where $\mathbf{K}^{t}=\mathbf{K}$.

The Lagrangian is given by

$$
L=\mathbf{x}^{t} \mathbf{A}^{t} \mathbf{A} \mathbf{x}+\mu\left(\mathbf{x}^{t} \mathbf{K} \mathbf{x}-1\right)
$$

and its derivatives are given by

$$
\begin{aligned}
& \frac{d L}{d \mathbf{x}}=2 \mathbf{A}^{t} \mathbf{A} \mathbf{x}+2 \mu \mathbf{K} \mathbf{x} \\
& \frac{d L}{d \mu}=\mathbf{x}^{t} \mathbf{K} \mathbf{x}-1
\end{aligned}
$$

Setting the derivatives to zero, gives

$$
\begin{array}{lll}
\frac{d L}{d \mathbf{x}}=\mathbf{0} & \Longrightarrow & \mathbf{A}^{t} \mathbf{A} \mathbf{x}=-\mu \mathbf{K} \mathbf{x} \\
\frac{d L}{d \mu}=\mathbf{0} & \Longrightarrow & \mathbf{x}^{t} \mathbf{K} \mathbf{x}=1
\end{array}
$$

Equation (17) means that $\mathbf{x}$ is a generalized eigenvector of $\left(\mathbf{A}^{t} \mathbf{A}, \mathbf{K}\right)$ with eigenvalue $-\mu$. Furthermore, by multiplying (17) on the left by $\mathbf{x}^{t}$, we get

$$
\mathbf{x}^{t} \mathbf{A}^{t} \mathbf{A} \mathbf{x}=-\mu \mathbf{x}^{t} \mathbf{K} \mathbf{x}=-\mu
$$

where we used (18) for the second equality. Note that the left-hand-side of (19) is the cost function $J(\mathbf{x})$; it can be concluded that $J(\mathbf{x})$ is minimized subject to $\mathbf{x}^{t} \mathbf{K x}=1$ by the generalized eigenvector corresponding to the minimum eigenvalue. 


\section{APPENDIX B. MINIMIZATION WITH WEIGHTED INNER PRODUCT - 2}

Consider the cost function

$$
J(\mathbf{x})=\mathbf{x}^{t} \mathbf{A}^{t} \mathbf{A} \mathbf{x}
$$

and a given symmetric positive semidefinite matrix $\mathbf{K}$. Suppose the columns of $\mathbf{B}$ are a partial set of generalized eigenvectors $\mathbf{B}$ of $\left(\mathbf{A}^{t} \mathbf{A}, \mathbf{K}\right)$,

$$
\mathbf{A}^{t} \mathbf{A B}=\mathbf{K B D}
$$

where $\mathbf{D}$ is a diagonal matrix. Suppose also that the columns of $\mathbf{B}$ are normalized to have unit norm with respect to $\mathbf{K}$, that is,

$$
\mathbf{B}^{t} \mathbf{K B}=\mathbf{I}
$$

Consider the constrained minimization problem:

$$
\min _{\mathbf{x}} J(\mathbf{x}) \quad \text { subject to } \quad \mathbf{B}^{t} \mathbf{K} \mathbf{x}=\mathbf{0} \quad \text { and } \quad \mathbf{x}^{t} \mathbf{K} \mathbf{x}=1 .
$$

The Lagrangian is given by

$$
L=\mathbf{x}^{t} \mathbf{A}^{t} \mathbf{A} \mathbf{x}+\boldsymbol{\mu}_{1}^{t} \mathbf{B}^{t} \mathbf{K} \mathbf{x}+\mu_{2}\left(\mathbf{x}^{t} \mathbf{K} \mathbf{x}-1\right)
$$

and its derivatives are given by

$$
\begin{aligned}
\frac{d L}{d \mathbf{x}} & =2 \mathbf{A}^{t} \mathbf{A} \mathbf{x}+\mathbf{K B} \boldsymbol{\mu}_{1}+2 \mu_{2} \mathbf{K} \mathbf{x} \\
\frac{d L}{d \boldsymbol{\mu}_{1}} & =\mathbf{B}^{t} \mathbf{K} \mathbf{x} \\
\frac{d L}{d \mu_{2}} & =\mathbf{x}^{t} \mathbf{K} \mathbf{x}-1
\end{aligned}
$$

Setting the derivatives to zero, gives

$$
\begin{array}{rll}
\frac{d L}{d \mathbf{x}}=\mathbf{0} & \Longrightarrow & \mathbf{A}^{t} \mathbf{A} \mathbf{x}=-\frac{1}{2} \mathbf{K B} \boldsymbol{\mu}_{1}-\mu_{2} \mathbf{K} \mathbf{x} \\
\frac{d L}{d \boldsymbol{\mu}_{1}}=\mathbf{0} & \Longrightarrow & \mathbf{B}^{t} \mathbf{K} \mathbf{x}=\mathbf{0} \\
\frac{d L}{d \mu_{2}}=\mathbf{0} & \Longrightarrow & \mathbf{x}^{t} \mathbf{K} \mathbf{x}=1
\end{array}
$$

Multiplying (25) on the left by $\mathbf{B}^{t}$ gives

$$
\mathbf{B}^{t} \mathbf{A}^{t} \mathbf{A} \mathbf{x}=-\frac{1}{2} \mathbf{B}^{t} \mathbf{K B} \boldsymbol{\mu}_{1}-\mu_{2} \mathbf{B}^{t} \mathbf{K} \mathbf{x}
$$

Using $\mathbf{B}^{t} \mathbf{K B}=\mathbf{I}$ and $\mathbf{B}^{t} \mathbf{K} \mathbf{x}=\mathbf{0}$ gives

$$
\mathbf{B}^{t} \mathbf{A}^{t} \mathbf{A} \mathbf{x}=-\frac{1}{2} \boldsymbol{\mu}_{1}
$$

From (20) we have $\mathbf{B}^{t} \mathbf{A}^{t} \mathbf{A}=\mathbf{D} \mathbf{B}^{t} \mathbf{K}$, so we get

$$
\mathbf{D B}^{t} \mathbf{K} \mathbf{x}=-\frac{1}{2} \boldsymbol{\mu}_{1}
$$

From (26) we have

$$
\boldsymbol{\mu}_{1}=\mathbf{0}
$$

so $(25)$ becomes

$$
\mathbf{A}^{t} \mathbf{A} \mathbf{x}=-\mu_{2} \mathbf{K} \mathbf{x}
$$

which implies that $\mathbf{x}$ is a generalized eigenvector of $\left(\mathbf{A}^{t} \mathbf{A}, \mathbf{K}\right)$ with eigenvalue $-\mu_{2}$. In addition, multiplying (28) on the right by $\mathbf{x}^{t}$ gives

$$
\mathbf{x}^{t} \mathbf{A}^{t} \mathbf{A} \mathbf{x}=-\mu_{2} \mathbf{x}^{t} \mathbf{K} \mathbf{x}=-\mu_{2} \quad \Longrightarrow \quad J(\mathbf{x})=-\mu_{2}
$$

where we used (27). Therefore, we should select the eigenvector (not already in $\mathbf{B}$ ) with minimum eigenvalue. 


\section{APPENDIX C. MINIMIZATION WITH ORTHOGONALITY CONSTRAINTS}

Consider the cost function

$$
J(\mathbf{x})=\mathbf{x}^{t} \mathbf{A}^{t} \mathbf{A} \mathbf{x}
$$

and a given symmetric positive semidefinite matrix $\mathbf{K}$. Consider the constrained minimization problem:

$$
\min _{\mathbf{x}} \mathbf{x}^{t} \mathbf{A}^{t} \mathbf{A} \mathbf{x} \quad \text { subject to } \quad \mathbf{B}^{t} \mathbf{x}=\mathbf{0} \quad \text { and } \quad \mathbf{x}^{t} \mathbf{K} \mathbf{x}=1 .
$$

Write $\mathbf{B}^{t} \mathbf{x}=\mathbf{0}$ as $\mathbf{x}=\mathbf{C} \mathbf{u}$ where $\mathbf{C}$ is an orthonormal basis for the null space of $\mathbf{B}^{t}$. Now we have an optimization problem over $\mathbf{u}$ and $\mathbf{C} \mathbf{u}$ then yields the optimal $\mathbf{x}$. Therefore, consider the constrained optimization problem

$$
\min _{\mathbf{u}} \mathbf{u}^{t} \mathbf{C}^{t} \mathbf{A}^{t} \mathbf{A C} \mathbf{u} \quad \text { subject to } \mathbf{u}^{t} \mathbf{C}^{t} \mathbf{K} \mathbf{C u}=1,
$$

or

$$
\min _{\mathbf{u}} \mathbf{u}^{t} \tilde{\mathbf{A}}^{t} \tilde{\mathbf{A}} \mathbf{u} \quad \text { subject to } \quad \mathbf{u}^{t} \tilde{\mathbf{K}} \mathbf{u}=1,
$$

where $\tilde{\mathbf{A}}:=\mathbf{A C}$ and $\tilde{\mathbf{K}}:=\mathbf{C}^{t} \mathbf{K} \mathbf{C}$. This is exactly the constrained optimization problem considered in Appendix A. Accordingly, the solution to (29) is given by $\mathbf{C u}$ where $\mathbf{u}$ is the generalized eigenvector of $\left(\mathbf{C}^{t} \mathbf{A}^{t} \mathbf{A C}, \mathbf{C}^{t} \mathbf{K} \mathbf{C}\right)$ with minimum eigenvalue.

\section{REFERENCES}

1. N. Ahmed, T. Natarajan, and K. R. Rao. Discrete cosine transform. IEEE Trans. on Computers, C-23(1):9093, January 1974.

2. B. Chen, H. Wang, and L. Cheng. Fast directional discrete cosine transform for image compression. Optical Engineering, 49(2), February 2010.

3. A. Dremeau, C. Herzet, C. Guillemot, and J.-J. Fuchs. Sparse optimization with directional DCT bases for image compression. In Proc. IEEE Int. Conf. Acoust., Speech, Signal Processing (ICASSP), 2010.

4. O. G. Sezer, O. Harmanci, and O. G. Guleryuz. Sparse orthonormal transforms for image compression. In Proc. IEEE Int. Conf. Image Processing, 2008.

5. G. Strang. The discrete cosine transform. SIAM Review, 41(1):135-147, 1999.

6. G. Strang. Bringing the SVD to life. SIAM News, 39(1), January/February 2006.

7. H. Xu, J. Xu, and F. Wu. Lifting-based directional DCT-like transform for image coding. IEEE Trans. Circuits Syst. Video Technol., 17(10):1325-1335, October 2007.

8. B. Zeng and J. Fu. Directional discrete cosine transforms - a new framework for image coding. IEEE Trans. Circuits Syst. Video Technol., 18(3):305-313, March 2008. 